\title{
Visual and anatomical results of surgery for long standing macular holes
}

\author{
Robert A H Scott, Eric Ezra, James F West, Z J Gregor
}

\begin{abstract}
Aims-To determine the visual and anatomical outcome of surgery for long standing idiopathic macular holes.

Methods-A retrospective review of 24 eyes of all 22 patients who underwent surgery for idiopathic full thickness macular holes (FTMH) symptomatic for between 1 and 3 years. Postoperative follow up was for 6 months. Preoperative and postoperative visual acuities were recorded as well as the presence of anatomical closure of the hole.

Results-The mean duration of symptoms was 18.21 (SD 5.42) months). Anatomical closure of the FTMH was achieved in 17 $(70.8 \%)$ of the eyes at 6 months. The $\operatorname{logMAR}$ acuity of the group where closure was achieved improved by a mean of 0.31 , equivalent to a change of Snellen acuity from $6 / 60$ to $6 / 29$. Where the hole remained open the acuity deteriorated by a mean $\log M A R$ of 0.11 lines, equivalent to a change of Snellen acuity from 6/60 to 5/60. Anatomical closure of the hole was associated with a significantly improved acuity over non-closure $(p<0.001)$. The degree of visual improvement was independent of the preoperative visual acuity (Spearman correlation coefficient $0.03, p=0.888$ ), though preoperative acuity was related to the final acuity (Spearman correlation coefficient $0.701, p<0.001)$. Over the study period, six patients required cataract surgery, one patient developed secondary glaucoma, and one a retinal detachment. Conclusions-Vitrectomy with intraocular gas tamponade and postoperative posturing is a well tolerated and effective intervention for long standing macular holes. Anatomical closure of the macular hole is associated with a significant improvement in visual acuity.

(Br f Ophthalmol 2000;84:150-153)
\end{abstract}

Vitrectomy with fluid-gas exchange is a well recognised and safe technique in the management of idiopathic full thickness macular holes (FTMH) ${ }^{1-3}$ Most studies demonstrate clear benefits if surgery is performed within 6 months of the onset of symptoms and an inverse relation between the duration of symptoms of FTMH and surgical success in terms of closure and visual acuity has been suggested. ${ }^{4}$

The success rate for long standing FTMH is less clear. ${ }^{6}$ There are situations where surgery may be considered for patients with long standing FTMH. For example, in patients pre- senting with bilateral FTMH of long duration or who have had successful surgery on one eye for a FTMH of recent onset on one eye and have a long standing FTMH in the fellow eye. In the latter situation, successful closure of a long standing FTMH may improve overall visual function and stereopsis. ${ }^{7}$ Our aim was to evaluate the visual and anatomical effects of standard vitreous surgery for long standing idiopathic FTMH with symptoms for more than 12 months and less than 36 months.

\section{Patients and methods}

This was a retrospective review of 24 eyes (right $54.2 \% /$ left $45.8 \%$ ) of 22 consecutive patients who underwent conventional surgery for idiopathic FTMH symptomatic between 12 and 36 months at Moorfields Eye Hospital from February 1995 to February 1997. The average duration of the macular hole was 18.21 (SD 5.42) months. One patient had a previous cataract extraction with posterior chamber lens implant procedure. The rest had no previous ocular diseases diagnosed.

The patients were examined preoperatively and at each subsequent visit with a record of the best corrected Snellen visual acuity, slit lamp biomicroscopy, tonometry, and a full fundus examination. The Snellen acuity was converted into a logarithm of the minimum angle of resolution ( $\log M A R)$ equivalent to aid statistical analysis. ${ }^{8}$ A diagnosis of a macular hole was confirmed on slit lamp biomicroscopy by the presence of a full thickness macular defect with a positive Watzke-Allen test. ${ }^{9}$ Posterior vitreous separation was diagnosed by noting the presence of a visible Weis's ring anterior to the optic nerve. The FTMH were graded according to the Gass classification. ${ }^{10}$ Fundus fluorescein angiography was performed where indicated.

A standard surgical technique was employed for all the procedures, which were carried out by three surgeons. The technique included vitrectomy, disinsertion of the posterior hyaloid face by suction at the edge of the optic disc, and fluid-gas (16\% perfluoropropane-air mixture) exchange. In all cases an attempt was made to search for epiretinal and internal limiting membranes from around the hole using a macular pick. In 15 cases $(62.5 \%)$ autologous serum was applied to the hole for 5 minutes before the fluid-gas exchange. ${ }^{11}$

Rigorous face down positioning for $50 \mathrm{~min}$ utes in the hour during the day and face down positioning during sleep was encouraged postoperatively for 12 days. After appropriate counselling and encouragement from a vitreoretinal specialist nurse combined with the use 
of a "Desktop Portal" head rest (Oakworks, USA) there was approximately 95\% compliance with posturing as measured by patient posture diaries (C Banks, personal communication, 1997).

The eyes were examined preoperatively and at 1 day, 2 weeks, 1 month, 3 months, and 6 months postoperatively. Additional visits were scheduled postoperatively if required. The results of the examinations were recorded on a prepared form for ease of data entry. The macular hole was graded as closed only if the edges were completely sealed to the retinal pigment epithelium. The macular hole was graded as open if any of the perimeter of the hole was elevated.

Comparisons of categorical data such as macular hole closure between subgroups of eyes were made using a two tailed Fisher's exact test. Comparisons between numerical variables were made using the Wilcoxon and Mann-Whitney U tests and correlation of data with Spearman's test.

\section{Results}

The mean age of the 22 patients was 68.3 years with range $42-82$ years. There was a female $(77.3 \%)$ preponderance with a female to male ratio of $3.4: 1$. Three $(12.5 \%)$ of the macular holes were graded as stage $2,11(45.8 \%)$ as stage 3 , and $10(41.7 \%)$ as stage 4 . All the macular holes had cystic changes noted around the edge.

Preoperatively, an abnormal macular surface reflex was noted in $21 / 24(87.5 \%)$ cases and the presence of epiretinal membrane (ERM) suspected in $16 / 24$ cases $(66.7 \%)$. An epiretinal membrane was peeled in $20 / 24(83.3 \%)$ cases. There were no peroperative complications. A postoperative rise in the IOP occurred in $7 / 24$ cases $(29.2 \%)$. This was transient in all but one case. All of the patients completed at least 6 months of follow up.

\section{ANATOMICAL OUTCOME}

After one operation 15/24 of the macular holes $(62.5 \%)$ were closed at the 1 month check. Of the remaining nine patients left with an open hole, two elected to have a further operation. The repeat procedure involved further peeling of epiretinal membranes and a fluid-gas exchange with 12 days of postoperative posturing. At 6 months there was anatomical closure in $17 / 24(70.8 \%)$ of the macular holes. The closure rate was not significantly affected by the use of autologous serum, it was 10/15 with serum and $7 / 9$ where no adjunct was employed (Fisher's exact test $\mathrm{p}=0.365$ ).

VISUAL ACUITY

The mean preoperative acuity was $\log$ MAR 0.83 (Snellen equivalent 6/41) with range 0.48-1.3 (618-3/60) and mode 1.0 (6/60). At 6 months there was an improvement in visual acuity to $\log$ MAR 0.65 (Snellen equivalent $6 / 27$ ); this was statistically significant (Wilcoxon matched pairs $\mathrm{p}<0.01$ ).

Sixteen eyes (66.7\%) had improved acuity by an average of $\log M A R \quad 0.33$, equivalent to a change in Snellen acuity from $6 / 60$ to $6 / 28$.

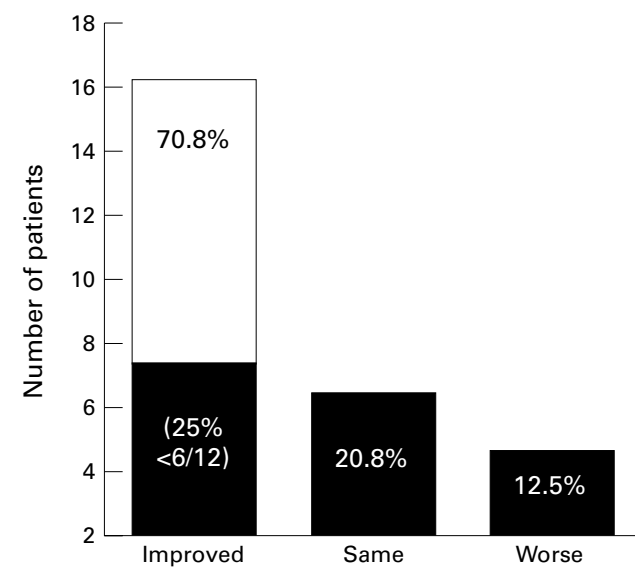

Figure 1 Histogram depicting the number and percentage of eyes according to visual outcome 6 months postoperatively.

The visual acuity did not change in five cases $(20.8 \%)$. Three cases $(12.5 \%)$ had a worse acuity with an average deterioration of logMAR 0.24, equivalent to a change in Snellen acuity from $6 / 60$ to approximately $3.5 / 60$. A Snellen acuity of $6 / 12$ or better was achieved in six eyes (25\%), as summarised in Figure 1.

In the group of 17 eyes where anatomical closure was attained the acuity significantly improved from the preoperative level by an average of $\log$ MAR 0.31 (Wilcoxon test $\mathrm{p}<0.001$ ), approximately equivalent to a change in Snellen acuity from 6/60 to 6/29. This group contained all of the eyes where improved acuity was observed. In one case there was no change; this eye had suffered a macular off-retinal detachment. The preoperative acuity in this group was not related to the total improvement of acuity (Spearman correlation coefficient $0.03, p=0.888$ ). It was closely related to the final postoperative acuity attained (Spearman correlation coefficient $0.701, \mathrm{p}<0.001$ ) (Fig 2). The eye that suffered a macular off-retinal detachment had no visual improvement despite closure of the FTMH.

The seven eyes where the FTMH failed to close at 6 months suffered a slight visual deterioration of on average $\log$ MAR 0.11 , approximately equivalent to a change in Snellen acuity from $6 / 60$ to $5 / 60$, this trend did not reach statistical significance (Wilcoxon test $\mathrm{p}=0.1$ ). Closure of the macular hole was associated with a significant improvement in visual acuity over non-closure (Mann-Whitney $U$ test $\mathrm{p}<0.001)$. The $\log$ MAR acuity of the groups

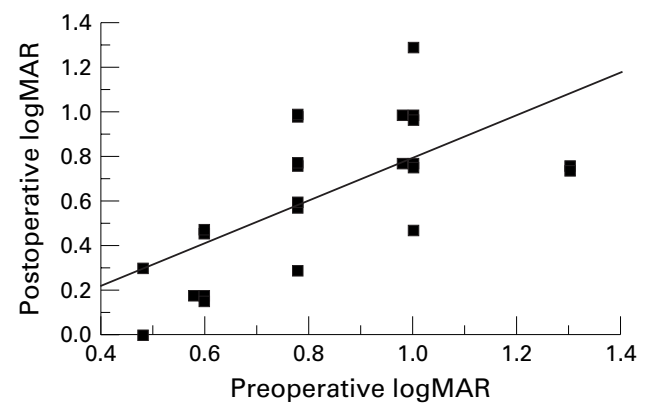

Figure 2 Relation between preoperative and postoperative $\log M A R$ acuity at 6 months. 


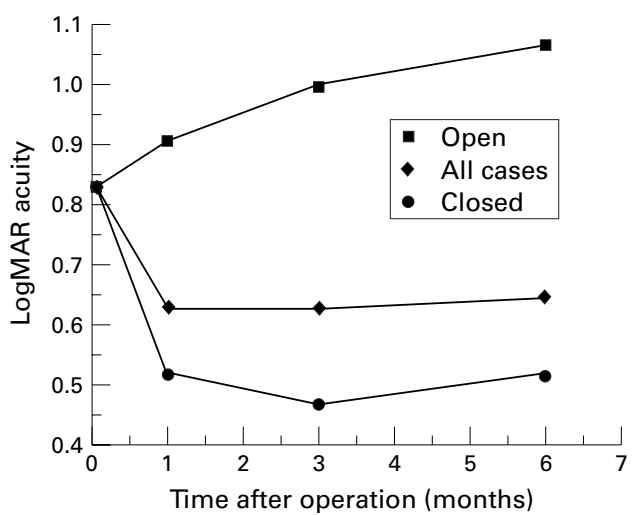

Figure 3 Postoperative logMAR acuity against time according to the anatomical result of surgery for FTMH.

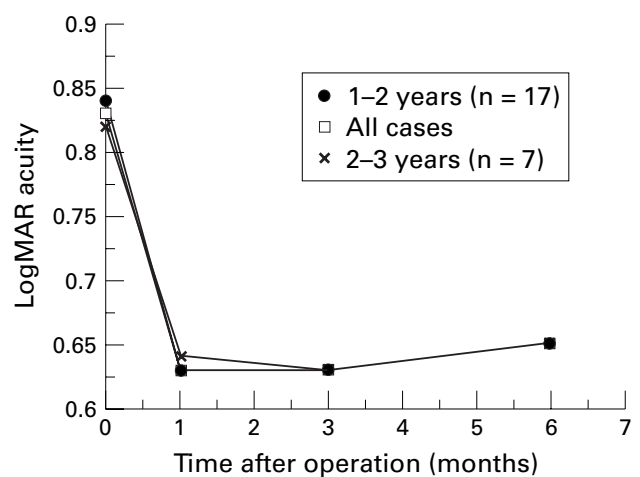

Figure 4 Postoperative $\log M A R$ acuity against time according to the duration of the FTMH.

according to closure of the FTMH is summarised in Figure 3.

DURATION OF SYMPTOMS

Seventeen $(70.8 \%)$ macular holes were symptomatic for between 1 and 2 years, the remaining seven $(29.2 \%)$ between 2 and 3 years. At 6 months there was no significant difference in the rate of anatomical closure between the two groups, $12 / 17(70.6 \%)$ versus 5/7 (71.4\%) respectively (Fisher's exact test $\mathrm{p}=0.9$ ). There was no significant difference in $\log$ MAR acuity preoperatively or any stage postoperatively (Mann-Whitney U test $\mathrm{p}>0.1$ ). No significant difference in the change of logMAR postoperatively was detected (Mann-Whitney U test $\mathrm{p}>0.1$ ), the results are summarised in Figure 4.

COMPLICATIONS

Twelve $(50 \%)$ of the eyes had observed progression of nuclear sclerotic lens opacity at 6 months. Cataract extraction was required in six cases $(12.5 \%)$ by this time. One case $(4.2 \%)$ developed secondary glaucoma requiring continued medical intervention. One of the eyes $(4.2 \%)$ required detachment surgery as a result of a superotemporal retinal break, 6 weeks after the macular hole surgery.

\section{Discussion}

In our study vitrectomy and fluid-gas exchange are a safe and successful treatment for FTMH symptomatic for over 12 months. Our initial closure rate of $62.5 \%$ improved to $70.8 \%$ at six months after repeat surgery in two cases.
Thompson et al achieved a closure rate of $79.2 \%$ in 24 macular holes of between 2 and 2.99 years' duration. This higher rate of closure was at least in part influenced by the inclusion of cases with shorter duration FTMH which had already undergone unsuccessful surgery and had a better surgical and visual outcome from reoperation than the primary long standing FTMH. ${ }^{6}$

Willis and Garcia-Cosio achieved an $85 \%$ closure rate in a group of 53 eyes with FTMH of between 6 and 216 months' duration. ${ }^{5}$ The authors did not mention either the average duration of the FTMH or the rate of reoperation among their cases, and the apparently high closure rate may have been due to the sample being skewed towards holes of shorter duration and thus a better prognosis, or by multiple operations when initial surgery had been unsuccessful.

Only two of the nine patients who did not have initial closure of the macular hole elected to undergo repeat surgery: this was successful in both cases. One patient whose macular hole remained open after a single procedure was quite satisfied with the surgical result as her uniocular distortion had been relieved, presumably by peeling an epiretinal membrane. It is possible that if the success rate of a repeat operation was emphasised at the initial interview that more patients would have had further surgery and improved the overall closure rate. ${ }^{12} 13$

There are no prospective data on the effect of autologous serum in macular hole surgery, though recent retrospective studies have been encouraging. ${ }^{11}{ }^{14}$ In our study, adjunctive autologous serum did not increase the chances of closure of the FTMH. Our impression is that meticulous membrane peeling and postoperative posturing with long acting intraocular gas tamponade maximises the chances of hole closure. ${ }^{51516}$ Epiretinal membranes are common in long standing macular holes and probably reduce the chances of closure. ${ }^{17}$ We were able to peel thickened epiretinal membranes in the majority $(83.3 \%)$ of our cases.

In our study the duration of the FTMH did not to affect either anatomical success or visual outcome after surgery. If there is a general cut off when surgery for very long standing FTMH exists it does not occur within this time span. Most of the long standing FTMH in our study were stage 3 or $4(87.5 \%)$ and were associated with a preretinal operculum. In a previous study approximately $39 \%$ of opercula were found to contain photoreceptor elements. In that study the presence of a full thickness operculum did not affect visual outcome. It is probable that the presence of such an operculum was also not important in the visual outcome of our study. ${ }^{18}$

The improvement of visual acuity on closure of the FTMH is probably related more to flattening of the area of detached retina around the hole. It is our clinical impression that this is often equivalent to several times the area of the hole itself. In the case of FTMH, photoreceptor degeneration of the detached retina does not appear to be advanced even after a 
prolonged period of detachment. This may be related to the shallowness of the detachment allowing at least some interaction between the retinal pigment epithelium and the photoreceptor layer. Preoperative evaluation of the macular region using high resolution imaging techniques such as optical coherence tomography would best explore this finding and might elucidate diagnostic criteria to identify patients with long standing FTMH who have a good visual prognosis from surgery. ${ }^{19}$

An improvement of Snellen acuity was recorded in $66.7 \%$ of our cases at 6 months. This occurred only with closure of the macular hole and on average approximated to an improvement of two Snellen acuity lines from $6 / 60$, a quarter of the eyes attained a Snellen acuity of $6 / 12$ or better. The final acuity was dependent on macular hole closure, the preoperative acuity, and the presence of any surgical complications. We did not evaluate subjective symptoms of distortion, or measure any changes in the overall quality of life from the operation. Closure of macular holes is known to improve stereopsis as well as reduce subjective symptoms of distortion and even if the measured acuity is not greatly increased, there may be an overall subjective visual improvement for the patient. ${ }^{717}$

Where the macular holes remained open there was slight reduction of visual acuity over the study period. This was largely due to the progression of lens opacity in $4 / 7(57.1 \%)$ of the eyes, none had cataract surgery. In addition to this the macular holes may have increased in size with time. ${ }^{17}{ }^{20-22}$

The main postoperative complication was lens opacity; $50 \%$ of the patients had documented progression of nuclear sclerosis over 6 months with $25 \%$ requiring cataract extraction. Cataracts are a well recognised complication of vitrectomy for macular holes with up to $76 \%$ of cases requiring extraction within 2 years of surgery. ${ }^{23}$ Cataracts reduce the postoperative acuity and should be operated on if significant. One of the eyes suffered a form of secondary chronic closed angle glaucoma. The single case of retinal detachment was probably due to a retinal break in the vicinity of an entry site.

\section{Summary}

In our study, patients presenting with macular hole symptoms of between 12 and 36 months have a reasonable chance of both anatomical and functional success from surgery. As part of the preoperative counselling they should be warned that more than one operation may be necessary to close the hole and that cataract surgery is likely to be required. The visual result is likely to depend on the initial acuity but if anatomical closure is attained the Snellen acuity is likely to improve.

1 Kelly NE, Wendel RT. Vitreous surgery for idiopathic macular holes. Results of a pilot study. Arch Ophthalmol 1991;109:654-9.

2 Freeman WR, Azen SP, Kim JW, et al. Prospective randomised trial of vitrectomy for stage 2 macular holes. Am 7 Ophthalmol 1996;121:605-14.

3 Freeman WR, Azen SP, Kim JW, et al. Vitrectomy for the reatment of full-thickness stage 3 or 4 macular holes. Results of a multicentred randomised clinical trial. The vitrectomy for treatment of macular hole study group. Arch Ophthalmol 1997;115:11-21.

4 Wendel RT, Patel AC, Kelly NE, et al. Vitreous surgery for macular holes. Ophthalmology 1993;100:1671-6.

5 Willis AW, Garcia-Cosio JF. Macular hole surgery. Comparison of long-standing versus recent macular holes. Ophthalmology 1996;103:1811-14.

6 Thompson JT, Sjaara RN, Lansing MB. The results of surgery for chronic macular holes. Retina 1997;17:493-501.

7 Eckardt U, Eckardt C. Binocular vision in idiopathic macular foramen. Pre- and postoperative findings. Ophthalmologe 1995;92:626-30.

8 Ferris FL, Bresnick GH, Bailey I. New visual acuity charts for clinical research. Am f Ophthalmol 1982;94:91-6.

9 Martinez J, Smiddy WE, Kim J, et al. Differentiating macular holes from macular pseudoholes. Am $\mathcal{f}$ Ophthalmol 1994;117:763-7.

10 Gass JD. Idiopathic senile macular hole. Its early stages and pathogenesis. Arch Ophthalmol 1988;105:371-6.

11 Wells JA, Gregor ZJ. Surgical treatment of full-thickness macular holes using autologous serum. Eye 1996;10:593-9.

12 Ezra E, Aylward GW, Gregor ZJ. Membranectomy and autologous serum for the treatment of full-thickness macular holes. Arch Ophthalmol 1997;115:1276-80.

13 Smiddy WE, Sjaarda RN, Glaser BM, et al. Reoperation after failed macular hole surgery. Retina 1996;16:13-18.

14 Monin C, Barale PO, Harrabi S, et al. Surgical treatment by vitrectomy and autologous serum of thirty-six idiopathic vitrectomy and autologous serum of thirty-six

15 Yooh HS, Brooks HL, Capone A, et al. Ultrastructural features of tissue removed during idiopathic macular hole surgery. Am f Ophthalmol 1996;122:67-75.

16 Thompson JT, Smiddy WE, Glaser BM, et al. Intraocular tamponade duration and success of macular hole surgery. Retina 1996;16:373-82.

17 Guyer DR, Green WR, de Bustros S, et al. Histopathologic features of idiopathic macular holes and cysts. Ophthalmology 1990;97:1045-51.

18 Ezra E, Munro PM, Charteris DG, et al. Macular hole opercula. Ultrastructural features and clinicopathological correlation. Arch Ophthalmol 1998;115:1381-7.

19 Ezra E, Munro PM, Charteris DG, et al. Macular hole opercula. Ultrastructural features and clinicopathological correlation (letter). Arch Ophthalmol 1998;116:965-6.

20 Smith RG, Hardman Lea SJ, Galloway NR. Visual performance in idiopathic macular holes. Eye 1990;4:190-4.

21 Hickichi T, Yoshida A, Akiba J, et al. Natural outcomes of stage 1, 2, 3, and 4 macular holes. $\mathrm{Br} f$ Ophthalmol 1995;79:517-20.

22 Leonard RE, Smiddy WE, Flynn HE. Visual acuity and macular hole size after unsuccessful macular hole surgery. Am $\mathcal{f}$ Ophthalmol 1997;123:84-9.

23 Thompson JT, Glaser BM, Sjaara RN, et al. Progression of nuclear sclerosis and long term visual results of vitrectomy with transforming growth factor beta-2 for macular holes. Am f Ophthalmol 1995;119:48-54. 